¿Qué es la historia del conocimiento? Cómo la información dispersa se ha convertido en saber consolidado a lo largo de la historia

Peter Burke,

TRAD. M.G. Ubaldini

Buenos Aires: Siglo Veintiuno

Editores, 2017

\title{
Una introducción \\ a la historia del conocimiento
}

\author{
Norma E. Levrand \\ Universidad Nacional del Litoral - \\ CONICET
}

Desde hace algunas décadas, el campo disciplinar de la historia se ha expandido. Se han producido desplazamientos en los intereses de las investigaciones históricas y una continua fragmentación de este universo. El texto de Peter Burke ofrece una introducción concienzuda a la historia del conocimiento, considerando sus antecedentes remotos y proveyendo un esquema de los conceptos, los problemas y los procesos que son observados por esta disciplina.

Peter Burke puede ser adscripto a la «nueva historia», movimiento polifacético nacido en Francia, que se define por su reacción a la «historia tradicional». Junto a Jacques Le Goff, Keith Thomas, Robert Darnton y Carlo Ginzburg, entre otros, forma parte de los académicos con más predicamento en la historia social y cultural. Su obra Historia social del conocimiento (dos tomos) es un elocuente reflejo de su trayectoria en la Historia Intelectual y la Historia Cultural.

El libro que reseńamos presenta de modo conciso algunos elementos de aquél, aunque invita al lector a leer estructuralmente el modo de hacer esta historia, dando cuenta de los núcleos principales que han abordado las obras de la temática. Para ello, el texto se organiza en cuatro capítulos en los cuales: a) se contextualiza la historia del conocimiento; b) se brinda un glosario de conceptos fundamentales de esta disciplina; c) se explican los procesos que determinan que algo pueda considerarse conocimiento y d) se enuncian los principales problemas que afronta la historia del conocimiento y algunas predicciones acerca de su futuro cercano. Además de las notas que ilustran la vasta bibliografía consultada por el autor, se ofrece al final del texto una línea del tiempo con una "cronología selecta» de estudios del conocimiento, desde el siglo XVII (la línea principia con el texto de Francis Bacon El avance del saber) hasta 2014 (la edición inglesa del texto corresponde al año 2015).

En el primer capítulo «Los conocimientos y sus historias», el autor da cuenta de que la historia del conocimiento es una disciplina nueva, aunque pueden encontrarse antecedentes desde el siglo XVII. Su auge se produce en el siglo XX, a partir de la confluencia con la sociología del conocimiento, la antropología, la

- Abogada, Doctora en Derecho. Docente de la asignatura Ciencia, Tecnología y Sociedad en la Universidad Nacional del Litoral. Docente del Seminario de Relaciones Laborales en la Universidad Autónoma de Entre Ríos. Miembro del proyecto CAI+D «Interacción de saberes: identificación y análisis de casos emergentes en relación con problemas sociales en la Argentina de principios de siglo XX|», dirigido por Oscar Vallejos. 
arqueología, la geografía y las ciencias políticas. La historia del conocimiento, en particular, se desarrolla a partir de la historia del libro y de la historia de la ciencia, indica Burke. Los Estudios Sociales de la Ciencia y la Tecnología también han realizado importantes aportes a este campo, a pesar de no estar mencionados en el texto. Desde la década de 1990, la historia del conocimiento ha ganado centralidad en Alemania, Francia y Estados Unidos. Dan cuenta de ello las obras colectivas, grupos académicos, cátedras, centros de investigación, cursos y congresos entre otras actividades académicas.

En este capítulo ya se observan ciertas advertencias del autor, referentes a considerar los conocimientos y saberes como un conjunto que muchas veces presenta dificultades para su clasificación, la necesidad de considerar las condiciones espaciales y temporales en la producción del conocimiento como así también la perspectiva de género. Finalmente, el autor invita a considerar los temas opuestos al conocimiento: el olvido y la ignorancia, que dan cuenta de los conocimientos perdidos y aquellos que se han rechazado de manera consciente.

En el segundo capítulo "Conceptos», presenta un glosario con los principales términos que se utilizan en los estudios del conocimiento. Como indica el autor, estos términos «son de utilidad no sólo para leer y escribir sobre la historia del conocimiento, sino también para reflexionar sobre ella» (Burke, 2017: 33). Si bien un poco árido para su lectura, este capítulo se presenta como fundamental para aquellos que desean internarse en los estudios de este ámbito.

En muchos de los conceptos presentados subyace una preocupación por la circulación de conocimientos y saberes. Burke explica que la producción de conocimientos e innovación está fuertemente influida por los individuos, grupos e instituciones que los producen como por los espacios geográficos en los cuales aparecen y se difunden. Advierte, así que «los conocimientos pueden ser plurales pero no iguales» (Ibid., 33). Asimismo, da cuenta del tránsito espacial y disciplinar de los conocimientos, como de la estabilización de los mismos en tradiciones y profesiones.

En el capítulo «Procesos», el autor presenta un perspicaz análisis de las prácticas de sistematización que conforman el proceso de elaboración y utilización del conocimiento. Señala que estas prácticas «dependen de la coyuntura, se realizan de acuerdo con diferentes reglas y diferentes tipos de apoyo en diferentes épocas y medios» (Burke, 2017: 69). Por ello, en la exposición de cada una de ellas, abundan los ejemplos que dan cuenta de esta diversidad. La finalidad de la sistematización es lograr la «objetividad» de conocimiento, a partir de cuatro etapas: 1) recopilación de la información, 2) análisis, 3) difusión y 4) uso del conocimiento.

$\mathrm{El}$ autor provee ejemplos de historias del conocimiento situadas en diferentes contextos espacio-temporales que revelan los condicionamientos culturales a la producción del conocimiento desde la etapa de la recopilación. Tanto las «formas» de reunir información como las herramientas para consultarla son mediadas por los fines, las disciplinas y las autoridades a las cuales responden. El análisis y la descripción del conocimiento, por su parte, tampoco se presentan como prácticas atemporales. El autor ofrece abundantes ejemplos del siglo XV en adelante que demuestran de qué modo la descripción, la cuantificación, la clasificación, la comparación y contraste, la interpretación, la verificación y la narración de los conocimientos se encuentran situadas y deben ser analizadas contextualizadamente.

La difusión del conocimiento habitualmente es descrita a partir de la «transferencia», no obstante el autor prefiere hablar de «circulación», verbo que exhibe 
las diferentes vías por las cuales el conocimiento se desplaza como así también las interferencias producidas por las traducciones, los malentendidos, las adaptaciones deliberadas y las adecuaciones culturales. Para terminar el capítulo, se ocupa de la utilización de los conocimientos describiendo los usos en los ámbitos religioso, político y económico como también la reutilización a partir de la apertura de archivos que benefició a las investigaciones académicas. Menos claro es el subtítulo de "utilización errada", en el cual se ejemplifica la utilización de conocimientos supuestamente universales y descontextualizados por parte del Estado y se hace un llamamiento a la valorización de los saberes locales y alternativos.

El último capítulo, «Problemas y perspectivas», presenta, por una parte, posiciones contradictorias al momento de hacer historia, y por otra, una sagaz mirada acerca del futuro de este campo. Algunas cuestiones son generales a la disciplina histórica, tales como el problema de la relación entre el conocimiento y la sociedad; la importancia relativa del cambio y la continuidad; las interpretaciones anacrónicas y los conflictos entre tradiciones que pueden conducir a un relativismo. Otras cuestiones son especialmente importantes para la historia del conocimiento, como la perspectiva triunfalista, que relega la pregunta por la ignorancia, los obstáculos al conocimiento y los conflictos entre conocimientos diferentes; o el constructivismo como perspectiva que, en casos extremos, no permite atender ciertas condiciones pre-existentes al proceso del conocimiento. La sobrevaloración de los agentes o actores individuales o las estructuras o sistemas sociales, tanto como la ausencia de una perspectiva de género en los estudios de historia del conocimiento también son señalados como problemas que deben ser enfrentados.

Burke augura una expansión en el lugar del conocimiento en la historia. Por una parte, considera que la historia del conocimiento será permeada por tres abordajes: un enfoque global, que hace hincapié en los encuentros, las colisiones, las traducciones y las hibridaciones; un enfoque social, que acusa mayor interés en los saberes cotidianos y los conocimientos tácitos y un interés por el muy largo plazo, que el autor denomina "historia cognitiva». Esta última es definida como una «historia de las mentalidades colectivas (...) que abarca milenios» o «una "arqueología del saber" en un sentido literal» (Burke, 2017: 162), es una historia preocupada por la producción y difusión de conocimiento desde el Homo habilis (2.700.000 años) hasta el Homo sapiens.

Más allá del cumplimiento de estos pronósticos, aquél que hace el autor al final del libro seguramente sea cierto: en las próximas décadas habrá un mayor interés por la historia del conocimiento. Por ello, celebramos la traducción de este texto informativo y esclarecedor acerca de un área de notable actualidad y que merece la atención de la academia local. Al respecto, pocas (o nulas) son las referencias del autor a investigaciones sobre la producción y circulación del conocimiento en Sudamérica y en Argentina en particular. Ello convoca a producir investigaciones en este área, a partir de los conceptos presentados, inicialmente, por el texto comentado. 
Del texto al metatexto. Estudios de literatura italiana y comparada

Daniel Alejandro Capano Buenos Aires: Biblos, 2018

\section{Destacado aporte en el campo de la italianística}

\author{
Nora Sforza \\ Universidad de Buenos Aires - \\ Universidad del Salvador
}

Daniel Capano abre su último libro, fruto de años de profundas lecturas y de intensas investigaciones en el campo de la italianística, de la narratología y de las literaturas comparadas con una frase que puede ser conmovedora, pero que al mismo tiempo nos interpela fuertemente, en una época en la que - acosados $\mathrm{y}$ atormentados por las modalidades con que las ciencias duras avalan o rechazan nuestros trabajos de investigación - estamos constreñidos a aceptar reglas de juego con las que no siempre acordamos. En efecto, los programas de investigación universitarios y las consabidas categorizaciones nos obligan a escribir ese artículo revelador que tendremos mucho cuidado en publicar en la revista especializada que nos brinde una mejor evaluación. Y tal vez todo esto nos hace olvidar la verdadera esencia de nuestro trabajo, vale decir ese objeto deleitoso junto con el que hemos crecido: el libro. Así nos dice Capano que «mientras las revistas trabajan sobre el olvido, el libro lo hace sobre el recuerdo» (Capano, 2018: 9). Pero el recuerdo que nos propone el ensayista a lo largo de estas páginas es también la propia declaración de la poética de una mente brillante que ha immagazzinato — permítaseme el uso del verbo en italiano- años y años de lecturas variadísimas atravesadas por su gran capacidad de teorizarlas y de compararlas de manera sobria y a la vez exquisita.

Así, cada uno de los cuatro apartados en que se divide el libro, precedido de un marco teórico abarcador que contribuye a ubicar las características epocales en que se sitúan los escritores estudiados y la problemática que desarrollan en sus textos, está atravesado por un particular modo de leer: un enfoque filológico es aplicado para explicar textos de la literatura bajomedieval y del primer Humanismo italianos; un análisis prevalentemente histórico-cultural es el que predomina en el estudio de las páginas del libro dedicadas al particular Romanticismo italiano, con especial énfasis en las obras de Manzoni y Leopardi; un recorrido por textos contemporáneos anclado fundamentalmente en la teoría literaria, la narratología, el psicoanálisis, la filosofía integran la tercera parte; y, por último, la «lectura comparada» que atraviesa los aportes que realiza el estudioso por diversos géneros de una literatura contemporánea donde, partiendo de su infinito conocimiento de las letras italianas se cruzan, se funden, se tocan obras italianas de los siglos XX y XXI

\footnotetext{
- Doctora en Letras (FFyL, UBA). Profesora Adjunta de Literatura Italiana y JTP Regular de Literatura Europea del Renacimiento (FFyL-UBA) Miembro del Comité Asesor de la Revista Modernidades de la UNC, del Comité de Redacción de Cartabianca (Roma - Florencia), Presidente de la Asociación de Docentes e Investigadores de Lengua y Cultura Italianas de la Argentina y codirectora de la colección de Clásicos Italianos de Colihue. Especialista en Teatro del Renacimiento italiano y en temas de Italianística.
} 
junto con los mitos griegos y variados textos de la tradición (y no solo) argentina, francesa y española. Si bien es cierto que todo el libro está surcado por interesantes sugestiones que logran hacernos repensar muchos supuestos de la crítica literaria, es evidente que en esta última parte dedicada a la literatura italiana comparada la erudición y las ideas de Daniel Capano alcanzan las cimas más altas.

En el apartado final, el investigador comienza por señalar el relieve que han alcanzado hoy los estudios comparados en el campo literario y precisar las nociones de comparación literaria y de literatura comparada. Realiza luego, un breve pero sustancial recorrido por la historia de la disciplina partiendo de la tradicional definición goethiana y revé su alcance en la actualidad. Repasa los conceptos formulados por la escuela francesa y los trabajos de Pierre Brunel, Yves Chevrel, René Étiemble y Daniel-Henri Pageaux.

Posteriormente, pasa revista a los estudios comparados en Italia, desde sus comienzos (De Sanctis), hasta nuestros días (Asor Rosa, Gnisci). Menciona además, las universidad italianas que incluyen en su plan de estudios la asignatura y las publicaciones periódicas que incorporan en sus páginas enfoques comparados.

Asimismo, transita por la escuela norteamericana y comenta los aportes de René Wellek, Anna Balakian, Harold Bloom, Werner Sollors y las investigaciones interdisciplinarias de George Steiner y Edward Said.

A modo de reflexión, Capano escribe:

Creo percibir en la actualidad la intensificación de los estudios comparados en tres zonas de indagación: la tematología, la imagología y la literatura de viajes (hodopórica), relacionadas con la difusión que alcanzan en este momento de la historia de la humanidad los intercambios culturales, el área de investigación multicultural. (193)

Tras lo cual el estudioso explora esas tres zonas que, de acuerdo con su opinión, despiertan en nuestro tiempo el interés de la literatura comparada.

Por otra parte, Capano hace notar que,

la apetencia de la literatura comparada es enorme, aspira a totalizar enfoques y a ampliar los horizontes del conocimiento, pero sin perder su verdadera esencia como es el análisis contrastivo, basamento y piedra angular de su especificidad, de tal modo contribuye a conocer con mayor hondura el proceso literario y por ende la naturaleza del hombre. (196)

Finalmente, ofrece una abarcadora articulación de intercambios y penetraciones mutuas entre diferentes textos de Ungaretti, Svevo, Eco, Calvino, Bufalino, Magris, Capriolo y Pavese, entre otros, con escritores de Espańa, Francia y Argentina. Así desfilan ante nosotros páginas cuyo contenido podemos compartir o con el que podemos disentir, pero que jamás nos dejan indiferentes, pues siempre nos interpelan y nos sacuden como llamándonos a buscar nuevos horizontes y, en definitiva, nuevos modos de afrontar lecturas. Todo ello, claro está acompañado de una abundante bibliografía clásica y actualizada.

Pero si bien, decíamos al principio, la presente recopilación puede ser leída como una suerte de declaración poética, hay otra cuestión de no menor importancia y es la que explicita el mismísimo autor en la Presentación: 
La recopilación aspira a completar un espacio crítico sobre la literatura y la cultura italianas, que se encuentra en nuestro medio desactualizado y disperso. [...] Si bien el libro — continúa el investigador- puede ser leído por un público que posea conocimientos básicos de literatura italiana, se destina en especial a docentes y alumnos universitarios en el ámbito hispanoamericano, dedicados a tal área de conocimiento. (11)

«Leer es comparar», nos dice. Pero creo que la conjunción de estos textos hacen algo más: dialogan entre sí y dialogan profundamente con nosotros mismos. Precisa el investigador:

El diálogo que establecen los textos entre sí a lo largo de la historia de la literatura resulta de vivo interés para críticos y estudiosos de las letras, pues ven en cada nueva publicación, a través de un dinámico entramado de similitudes y diferencias, el producto de una amalgama entre los influjos que recibe el autor y las modificaciones que le imprime. Quizá, la originalidad surja de la justa dosis entre ambos componentes, pues las literaturas se configuran como un palimpsesto en el que el escritor escribe sobre lo que otros ya han escrito. (189-90)

Como lo hicieran los humanistas de los albores de la Modernidad clásica, también nuestro querido maestro no se queda con nada para sí y, dialogando desde y con el texto, todo lo comparte con nosotros, sus lectores. 